\title{
Las tecnologías de la información y comunicación como herramienta de inclusión social en la Educación Superior
}

\section{Information and communication technologies as a tool of social inclusion in Higher Education}

\author{
Nelson Mariscal Huacón ${ }^{1}$, Jhonathan Castro Rumazo ${ }^{1}$, César Caicedo-Camposano ${ }^{2}$ y Ariana Cruz-Posligua $^{2}$ \\ ${ }^{1}$ Instituto Tecnológico Superior Babahoyo, Ecuador \\ ${ }^{2}$ Universidad Nacional Autónoma de Los Andes, Ext. Babahoyo, Ecuador \\ *ing.nelsonmariscal@hotmail.com
}

DOI: https://doi.org/10.26871/killkana_social.v4i1.632

\begin{abstract}
Resumen
La investigación tiene como objetivo analizar Las Tecnologías de la Información y la comunicación como herramienta de inclusión social en la educación superior, siendo aplicada a diez cursos del Instituto Tecnológico Superior Babahoyo en el periodo 2017, donde existen estudiantes con algún tipo de discapacidad. A conveniencia de los investigadores se toma una muestra de 10 personas con discapacidad con quienes se interactuó dos veces por semana con el uso de internet en sus dispositivos móviles y laptops. Luego mediante la encuesta aplicada a la muestra nos refleja la importancia del uso tecnológico como parte fundamental en el proceso de educación, donde todos los elementos arrojan un $93 \%$ como factible el uso de las TIC. Uno de los hallazgos más relevantes fue identificar los recursos de TIC que poseen en los hogares los estudiantes con discapacidad en la encuesta y fue visible que la laptop es el dispositivo electrónico más utilizado. Estos datos muestran con certeza que el impacto de las TIC en el desarrollo integral de los estudiantes con discapacidad podría ser trascendental si fuese impartido de forma cotidiana y frecuente, tanto en contextos familiares como en contextos educativos y sociales. No obstante la investigación conlleva a que se enfatice el uso de las TIC en el salón de clases sin distinción, ni discriminación, más bien de forma inclusiva.
\end{abstract}

Palabras clave: educación, tic, inclusión, social, superior.

\begin{abstract}
The research aims to analyze Information and Communication Technologies as a tool for social inclusion in higher education, being applied to ten courses of the Higher Technological Institute Babahoyo in the period 2017, where there are students with some type of disability. At the convenience of the researchers, a sample of 10 people with disabilities is taken with whom they interacted twice a week with the use of the internet on their mobile devices and laptops. Then, through the survey applied to the sample, it reflects the importance of technological use as a fundamental part in the education process, where all the elements show $93 \%$ as feasible the use of TIC. One of the most relevant findings was to identify the TIC resources that students with disabilities have in the household and it was visible that the cell phone is one of the main resources they have, followed by the television, personal computer and the Internet, with The minimum result was the Tablet and laptop. These data show with certainty that the impact of ICT in the integral development of students with disabilities could be transcendental if it were taught on a daily and frequent basis, both in family contexts and in educational and social contexts. However, the research leads to emphasis on the use of ICT in the classroom without distinction, or discrimination, rather in an inclusive manner.
\end{abstract}

Keywords: education, tic, inclusion, social, superior.

\section{Introducción}

Las entidades de Educación Superior constituyen en la actualidad contextos caracterizados por la inmensa diversidad de sus estudiantes en el ámbito de discapacidades diferentes, siendo una población estudiantil heterogénea. Cuando se habla de reforma educativa se alude a las decisiones que toma un Estado ecuatoriano con el fin de orientar políticas educativas para superar las deficiencias detectadas en un sistema con resultados insatisfactorios en términos de calidad y eficiencia. La Educación Especial es un servicio diferencial en el contexto de la Educación Regular. Atiende a niños, adolescentes, jóvenes y adultos con características biológicas, psíquicas y socio-culturales diferentes, como consecuencia de antecedentes patológicos o clínicos, o de privaciones socioeconómicas y culturales. 
No es una instancia marginal, en virtud de que su función es incorporar al alumno, en el menor tiempo posible, a la educación común (Álvarez y Mayo, 2009).

En el artículo 71 de la Ley Orgánica de Educación Superior manifiesta que: "Principio de igualdad de oportunidades.- El principio de igualdad de oportunidades consiste en garantizar a todos los actores del Sistema de Educación Superior las mismas posibilidades en el acceso, permanencia, movilidad y egreso del sistema, sin discriminación de género, credo, orientación sexual, etnia, cultura, preferencia política, condición socioeconómica o discapacidad.

Las instituciones que conforman el Sistema de Educación Superior propenderán por los medios a su alcance que, se cumpla en favor de los migrantes el principio de igualdad de oportunidades.

Se promoverá dentro de las instituciones del Sistema de Educación Superior el acceso para personas con discapacidad bajo las condiciones de calidad, pertinencia y regulaciones contempladas en la presente Ley y su Reglamento. El Consejo de Educación Superior, velará por el cumplimiento de esta disposición." (Consejo de Educación Superior, 2010).

La educación es un derecho de las personas a lo largo de su vida y un deber ineludible e inexcusable del Estado. Constituye un área prioritaria de la política pública y de la inversión estatal, garantía de la igualdad e inclusión social y condición indispensable para el buen vivir. Las personas, las familias y la sociedad tienen el derecho y la responsabilidad de participar en el proceso educativo. La educación se centrará en el ser humano y garantizará su desarrollo holístico, en el marco del respeto a los derechos humanos, al medio ambiente sustentable y a la democracia; será participativa, obligatoria, intercultural, democrática, incluyente y diversa, de calidad y calidez; impulsará la equidad de género, la justicia, la solidaridad y la paz; estimulará el sentido crítico, el arte y la cultura física, la iniciativa individual y comunitaria, y el desarrollo de competencias y capacidades para crear y trabajar. (Asamblea Constituyente, 2008).

El uso de las Tecnologías de la Información y la Comunicación (TIC) que de forma habitual que desarrolla hoy en día dentro de las clases, mismas que se encuentran presentes en nuestro mundo, forman parte de nuestra cultura, de nuestra vida. Como señala Sancho Gil (2006), las personas que viven en lugares donde las TIC han eclosionado con celeridad no encuentran dificultades para apreciar cómo éstas han transformado multitud de aspectos de su vida cotidiana.

Dentro de este proceso de reinvención de las universidades, las TIC cobran un importante protagonismo, conformándose como elementos esenciales para el desarrollo de la flexibilidad organizativa en la enseñanza-aprendizaje y el desarrollo de nuevas sinergias que inserten plenamente a la universidad en el actual entramado de las sociedades del conocimiento. Las experiencias desarrolladas en este sentido auguran un futuro prometedor para aquellas instituciones de educación superior que integren, de forma efectiva, las TIC en sus procesos y estructuras, puesto que de forma general podemos indicar que:

- La introducción y el uso de las TIC en los procesos de enseñanza-aprendizaje y de gestión en la educación superior ha supuesto una transformación institucional orientada a dar respuesta a las necesidades demandadas por las sociedades del conocimiento, siendo una herramienta muy útil para dicho proceso.

- Las nuevas exigencias en la educación superior se centran en la mejora del proceso educativo $\mathrm{y}$, en este sentido, la integración de las TIC facilita aspectos relacionados con la mejora del trabajo individual, la autonomía a estudiantes, la facilidad para el desarrollo de trabajos en equipo y colaborativos, la posibilidad de modificar y adaptar los métodos de evaluación y la interacción bidireccional entre el profesorado y el alumnado.

\section{Materiales y Métodos}

El tipo de investigación utilizada fue de campo, se realizó en la ciudad de Babahoyo en el Instituto Tecnológico Superior Babahoyo, ubicado en la avenida Enrique Ponce Luque, siendo esta una institución pública donde se prestaron todas las facilidades mediante el apoyo de docentes y estudiantes para poder aplicar las respectivas encuestas. Los instrumentos fueron aplicados a los cincuenta docentes todos estos de las diferentes carreras ofrecidas por la IES, también se realizó una ficha valorativa electrónica, aplicada alrededor de mil ciento diecisiete estudiantes que poseen alguna discapacidad o necesidad. Los resultados obtenidos hacen referencia a dos instantes: la primera al conocimiento de conceptos de inclusión social y la otra hace referencia a las diferentes herramientas TIC en las cuales un estudiante utiliza para su aprendizaje.

\section{Resultados}

Una de las aristas en la inclusión social es se da para las personas con algún tipo de discapacidad, en la que conlleva el uso de herramientas tecnológicas para poder participar en el proceso educativo. Esta investigación se realizó a todos los estudiantes de la IES Babahoyo para el efecto se realizó la encuesta ver figuras 1 y 2. 


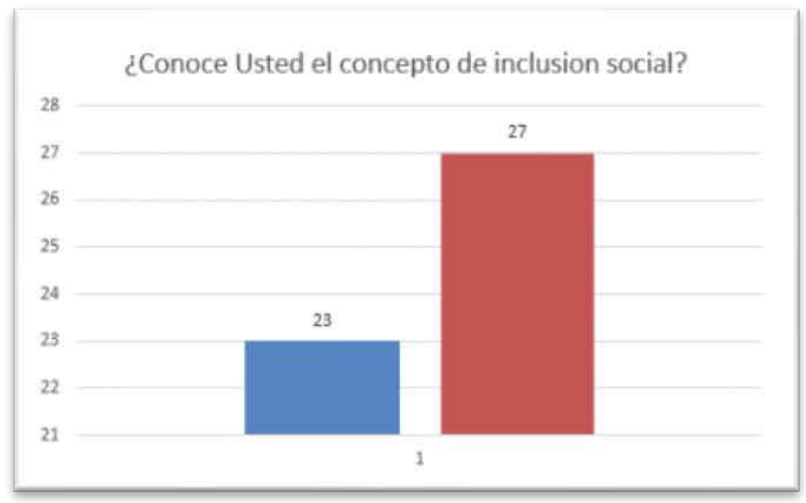

Figura 1: ¿Conoce Usted el concepto de inclusión social?

Fuente: Instituto Tecnológico Superior Babahoyo

Los resultados demuestran que los docentes en un porcentaje considerable conocen el concepto de inclusión, lo que hace posible poder resolver una problemática que aqueja a la sociedad. Una vez realizado el análisis en las encuesta se indica que en la figura 1 donde se realizó la encuesta a docentes con la pregunta ¿Conoce Usted el concepto de inclusión social?, podemos denotar el desconocimiento del término inclusión, lo que conlleva a que se debe profundizar más en este ámbito para poder erradicar las limitaciones que atraviesan los estudiantes con diferentes necesidades, que en su mayoría son ocasionadas por poseer algún tipo de discapacidad.

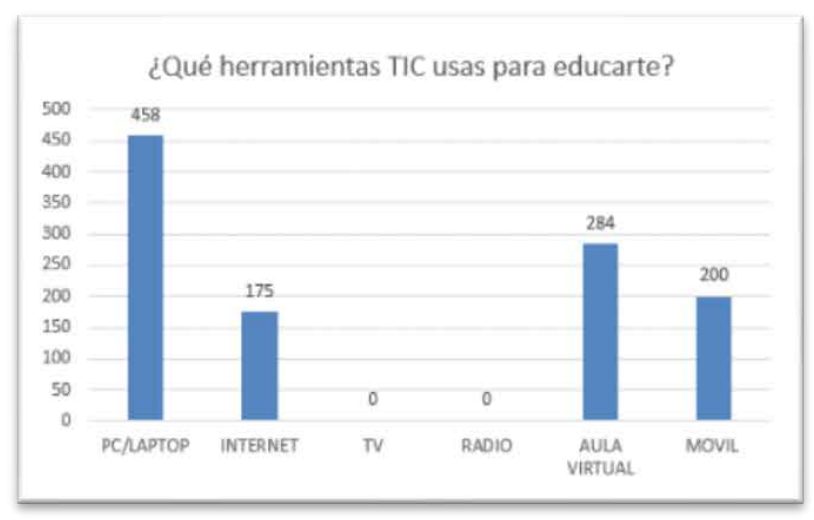

Figura 2: ¿Qué herramientas TIC usas para estudiar?

Fuente: Instituto Tecnológico Superior Babahoyo

Para este grafico los resultados denotan que una cantidad de estudiantes manejan herramientas tecnológicas lo que se hace imprescindible su aplicación. En la figura 2 cuya pregunta hace referencia al tipo de TIC que utiliza un estudiante para educarse, nos denota que en su mayoría tienen acceso a diferentes herramientas, mismas que son imprescindibles al momento de adquirir un conocimiento, haciendo énfasis que sin duda alguna en una población pequeña poseen alguna discapacidad como lo indica la figura 3 .

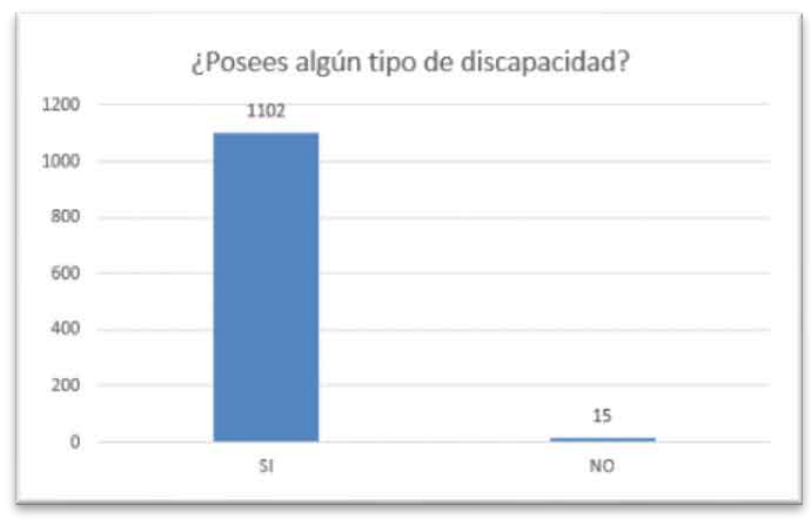

Figura 3: ¿Posee algún tipo de discapacidad?

Fuente: Instituto Tecnológico Superior Babahoyo

En la figura 3 podemos verificar que la población estudiantil en minoría hay personas con discapacidad lo que nos indica que para ellos es necesario el uso de una herramienta TIC para poder estudiar.

\section{Discusión de resultados}

Esta investigación tiene a bien puntualizar aspectos importantes sobre la problemática basada en los datos recopilados en la entrevista y dar a conocer a las entidades de educación superior las necesidades de los docentes en lo concerniente a educación especial, los tipos de necesidades que los estudiantes afrontan para que sean incluidos socialmente en el quehacer educativo, cuáles son sus requerimientos para poder brindarles un buen aprendizaje. Es menester indicar algunos puntos que deben puntos que deben ser implementados para mejorar el proceso de educación en las IES:

- Implantación de equipos tecnológicos acorde a las diferentes necesidades y en especial cuando se trata sobre discapacidad, ya sea esta fisca o intelectual, par de esta manera poder contribuir con el crecimiento educativo del estudiante.

- Las herramientas a utilizar son muchas por tal motivo se debe estar capacitado para poder ejercerlas en la doctrina educativa.

- Existen estudiantes que son de escasos recursos económicos, por lo que en ocasiones es imposible poder utilizar un equipo tecnológico limitándolo a no poder realizar alguna actividad académica.

- Los estudiantes en su mayoría manejan una herramienta tic, pero se debe dar el valor agregado de ¿Cómo de usarse?.

- Es imprescindible hacer énfasis a la relevancia que tiene una necesidad, para que en base a ella se puedan tomar los correctivos respectivos.

\section{Conclusiones}

En los resultados obtenidos tanto en la encuesta como en la ficha valorativa, se puedo identificar que en los 
hogares de estudiantes de educación superior poseen como primer elemento tecnológico una PC o Laptop lo que se presume que si están en la capacidad de superar la brecha digital y acercarlos más al mundo de la información y comunicación, es por tal motivo, que se vuelve sumamente importante utilizar las TIC como herramienta en el proceso de enseñanza-aprendizaje en los actores de la educación superior. De tal manera de incidir de forma indirecta en la vinculación de programas y espacios que utilicen como eje transversal las TIC.

La vinculación a un ambiente de aprendizaje mediado por las TIC, garantizó, en primera instancia, una estrategia para disminuir la brecha digital de los adolescentes con discapacidad, ya que se ha confirmado el postulado de Rodríguez (2012), es por esta razón que debemos crear condiciones para que las personas con menor posibilidad de acceso y uso de las TIC puedan mejorar su condición de vida.

Todo este contexto investigativo deja como evidencia que los estudiantes con discapacidad presentan un mayor interés en el proceso de enseñanza - aprendizaje, asumiendo un rol más activo y productivo al beneficiarse con el uso de herramientas tic sintiéndose motivados

\section{Referencias}

Álvarez, R. B., y Mayo, I. C. (2009). Las tecnologías de la información y la comunicación en la educación superior. Revista Iberoamericana de Educación/Revista Ibero-americana de Educação(50/7), 10.

Asamblea Constituyente. (2008). Constitución de la república del ecuador. Montecristi.

Consejo de Educación Superior. (2010). Ley orgánica de educación superior (loes). Gaceta del CES. Descargado de http://www.ces.gob .ec/gaceta-oficial/reglamentos

Durán, T. (2010). Importancia del proceso de aprendizaje y sus implicaciones en la educación del siglo xxi. Revista electrónica de pedagogía ODISEO, 7(14), 1870-1477.

European Agency for Development in Special Needs Education. (2013). Tecnologías de la información y la comunicación para la inclusión avances $y$ oportunidades en los países europeos. Descargado de https://www.european -agency.org/sites/default/files/ ICT_for_Inclusion-ES.pdf

Fernández-Cárdenas, J. M. (2013). El habla en interacción y la calidad educativa: los retos de la construcción de conocimiento disciplinar en ambientes mediados por tecnología digital. Revista mexicana de investigación educativa, 18(56), 223-248.

Liscano, R. E. G., Liscano, V. A. G., y Solano, M. E. L. (2017). Las nuevas reformas educativas en el ecuador y su aplicación en educación especial. Journal of Science and Research: Revista Ciencia e Investigación, 2(5), 10-13.

Montenegro, R., y Tigre, M. (2013). Rol de los docentes en la inclusión educativa (Tesis de Master no publicada). (Monografía previa a la obtención del título de Licenciada en Psicología Educativa en la especialización de Psicología Infantil. Cuenca: Universidad de Cuenca, Facultad de Psicología)

Trías, F., y Ardans, E. (2004). Las tecnologías de la información y la comunicación en la formación docente: Guía de planificación. Unesco.

Recibido: 5 de marzo de 2019

Aceptado: 13 de diciembre de 2019 